\title{
Lipid-based nanocarriers for oral delivery of peptides
}

\author{
Camille Dumont* \\ R\&D Pharma, Gattefossé SAS, 36 chemin de Genas, 69800 Saint-Priest, France
}

Received 1 September 2021 - Accepted 21 October 2021

\begin{abstract}
Therapeutic peptides can treat a wide variety of diseases with selective and potent action. Their oral bioavailability is strongly limited by an important proteolytic activity in the intestinal lumen and poor permeation across the intestinal border. We have evaluated the capacity of solid lipid nanoparticles (SLN) and nanostructured lipid carriers (NLC) to overcome both oral bioavailability limiting aspects, using leuprolide (LEU) as model peptide. Lipidization of LEU by formation of a hydrophobic ion pair (HIP) with sodium docusate enables a significant increase of peptide encapsulation efficiency in both SLN and NLC. The nanocarriers, obtained by high-pressure homogenization, measured $120 \mathrm{~nm}$ and were platelet shaped. Regarding the protective effect towards proteolytic degradation, only NLC maintained LEU integrity in presence of trypsin. Intestinal transport, evaluated on Caco-2 (enterocyte-like model) and Caco-2/HT29MTX (mucin-secreting model) monolayers, showed nanocarriers internalization by enterocytes but no improvement of LEU permeability. Indeed, the combination of nanoparticles platelet-shape with the poor stability of the HIP in the transport medium induces a high burst release of the peptide, limiting nanoparticles capacity to transport LEU across the intestinal border. Stability of peptide lipidization needs to be improved to withstand biorelevant medium to benefit from the advantages of encapsulation in solid lipid nanocarriers and consequently improve their oral bioavailability.
\end{abstract}

Keywords: peptides / oral delivery / solid lipid nanoparticles / nanostructured lipid carriers / hydrophobic ion pair / proteases / intestinal permeability / formulation

Résumé - Vecteurs lipidiques pour l'administration orale de peptides. Les peptides thérapeutiques peuvent traiter de nombreuses pathologies de manière efficace et sélective. Leur biodisponibilité orale est limitée par une forte dégradation liée à l'action de protéases dans le lumen intestinal et par une faible absorption par la barrière intestinale. Nous avons évalué la capacité des nanoparticules lipidiques solides (SLN) et des vecteurs lipidiques nanostructurés (NLC) à augmenter la biodisponibilité orale d'un peptide modèle : le leuprolide (LEU). L'augmentation de sa lipophilie par formation d'une paire d'ions hydrophobe (HIP) avec le docusate de sodium permet d'augmenter significativement le taux d'encapsulation du LEU dans les nanovecteurs. Ceux-ci sont obtenus par homogénéisation haute pression avec une taille de $120 \mathrm{~nm}$ et une structure en plaquettes. Vis-à-vis de la dégradation protéolytique, les NLC montrent une protection significative du LEU en présence de trypsine. L'évaluation du passage intestinal sur des monocouches de Caco-2 (modèle entérocyte) et de Caco-2/HT29-MTX (modèle sécrétant des mucines) révèle une internalisation des nanovecteurs mais aucune amélioration de l'absorption. En effet, la morphologie en plaquettes des SLN et NLC associée à la faible stabilité de l'HIP dans le milieu provoquent une libération importante du LEU, annulant la capacité de transport des nanovecteurs à travers la barrière intestinale. Il convient d'améliorer la stabilité de l'HIP pour augmenter la biodisponibilité orale des peptides via l'encapsulation dans des nanovecteurs lipidiques solides.

Mots clés : peptides / oral / nanoparticules lipidiques solides / vecteurs lipidiques nanostructures / paire d'ions hydrophobe / protéases / perméabilité intestinale / formulation

\footnotetext{
Contribution to the Topical Issue "Bioactive lipids and lipid droplets: green ressources for food and health / Lipides et gouttelettes lipidiques bioactifs : des ressources vertes pour l'alimentation et la santé".

*Correspondence: cdumont@gattefosse.com
} 


\section{Context}

\subsection{Peptides and associated limitations for oral delivery}

Peptides display high specificity and selectivity for some drug targets, offering pharmacological advantages over small molecules by increasing the capacity of action while reducing secondary effects (Craik et al., 2013). On a physico-chemical point of view, these molecules are generally hydrophilic and exhibit a high molecular weight, between 500 and $5000 \mathrm{kDa}$.

Most therapeutic peptides are delivered through parenteral administration. Depending on the administered drugs, these injections may be painful and frequently repeated to overcome the poor viability of therapeutic peptides in human physiological fluids (Morishita and Peppas, 2006). Administrating these drugs would considerably improve patients' comfort and compliance to medications as well as reducing the costs of treatments.

Lot of research is currently done on oral delivery of peptides. However, although 60 peptides were approved by the FDA in 2016 , only 12 peptides are currently marketed as oral dosage forms ( 2 being approved in 2019 and 2020 respectively) and, among them, only 6 peptides are intended to reach the systemic circulation, even if their oral bioavailability barely exceeds 1\%. (Aguirre et al., 2016; Richard, 2017).

\subsection{Oral peptide delivery: a harsh journey throughout the gastro-intestinal tract}

Low oral bioavailability of peptides is the result of multiple factors which can be perceived as a succession of barriers in the gastro-intestinal tract.

The first barrier is constituted by the acidic environment of the stomach which can alter the stability of peptides and consequently result in a loss of therapeutic activity (Leonaviciute and Bernkop-Schnürch, 2015). Furthermore, pepsins, proteases located in the stomach, can fragment administered peptides into smaller peptides and/or amino acids by cleaving functions of specific amino acids. It is however possible to protect the drugs from this harsh environment by using gastro-resistant capsules. These devices are covered by an enteric coating which only dissolves and releases its content when the $\mathrm{pH}$ increases.

When peptides reach the upper part of the intestine, they encounter the second part of the enzymatic barrier with trypsin, $\alpha$-chymotrypsin and elastase, playing a prominent role in peptide degradation. For peptide exhibiting disulfide bridges, thiol-disulfide exchange reactions may be induced by the presence of reduced glutathione.

The oral bioavailability of peptides is not only limited by enzymatic degradation but also by poor intestinal permeation. Indeed, to reach the systemic circulation, peptides must first permeate across the mucus layer which covers the surface of the intestinal border. This positively charged gel layer prevents the permeation of xenobiotics and therefore may limit the permeation of therapeutic peptides. Finally, the intestinal epithelium represents an additional barrier to high peptide systemic uptake. It is mainly composed of enterocytes whose lipophilic plasma cell membranes limit the absorption of hydrophilic peptides. These cells are separated by narrow intercellular spaces into which tight junctions prevent the permeation of large macromolecules (Malhaire et al., 2016).

\subsection{Advanced drug delivery systems: lipid-based nanocarriers}

Among the numerous strategies currently considered by formulators to increase oral peptide bioavailability, the use of nanocarriers (objects with a size $<1000 \mathrm{~nm}$ ) appear as a promising approach, enabling enhanced distribution of the loaded drug at the surface of the intestine and promoting intestinal absorption (Dumont et al., 2018). Among the different types of nanostructures, lipid-based nanocarriers are biocompatible, biodegradable and their lipophilic nature may help increasing the transcellular transport of loaded drugs. Several lipid-based nanocarriers are reported in the literature: nanoemulsions, liposomes, nanocapsules, self-emulsifying drug delivery systems (SEDDS) and solid lipid nanocarriers. These two latter systems were identified as the most suitable to increase the oral bioavailability of therapeutic peptides. Indeed, SEDDS were shown to protect encapsulated peptides from intestinal proteases activity (Hetényi et al., 2017) and improve mucus permeation (Griesser et al., 2018). The aim of the study presented in this article was to evaluate the capacity of the second type of promising particles, solid lipid nanocarriers, to improve oral bioavailability of model peptides.

Solid lipid nanocarriers refers to two types of nanoparticles: solid lipid nanoparticles (SLN), composed of solid lipid excipients at room and body temperature and stabilized by surfactants, or nanostructured lipid carriers (NLC), in which the solid lipid excipients is supplemented by a liquid lipid fraction (Müller, 2011). The addition of liquid fraction is said to enhance the stability of the carriers by increasing the mobility between the alkyl chains, thus reducing the risk of drug expulsion by modification of the crystal lattice over time.

\section{Design and evaluation of peptide loaded solid lipid nanocarriers}

\subsection{Peptide lipidization: formation of Hydrophobic Ion Pairs}

Several strategies can be considered to increase the lipophilicity (and affinity for lipid-based carriers) of therapeutic peptides (Dumont et al., 2018). Among them, the formation of Hydrophobic Ion Pairs (HIP) is particularly interesting on a regulatory point of view as the operation is reversible and does not affect the integrity of the drug.

To form HIP, therapeutic peptides containing at least one ionizable function are ionized in specific $\mathrm{pH}$ conditions and mixed with ionic surfactant solutions. The samples are centrifuged to separate the supernatant from the formed complexes which are then freeze-dried to be further encapsulated in the lipid-based nanocarriers. Precipitation efficiency can be calculated by quantifying the unprecipitated peptide in the supernatant. 

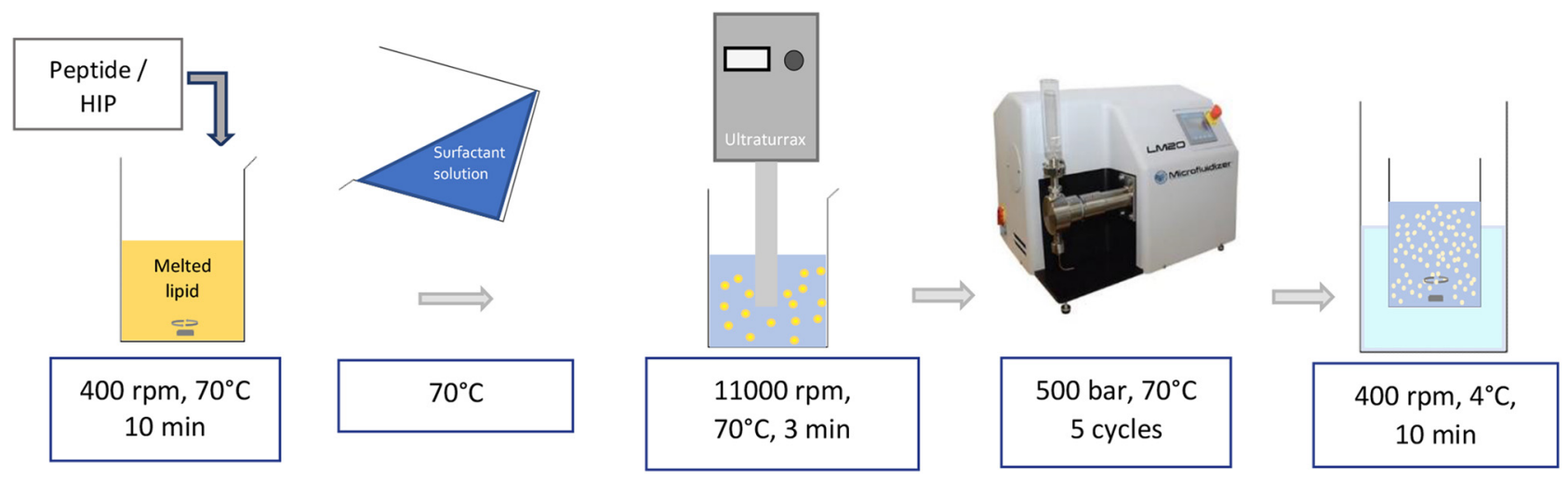

Fig. 1. Developed protocol to formulate peptide-loaded solid lipid nanocarriers.

HIP: hydrophobic ion pair.

Two model peptides were used in our study: Leuprolide used in the treatment of endometriosis, exhibiting 2 ionizable functions and a $\log \mathrm{P}$ value of -2 , and desmopressin, an antidiuretic hormone, exhibiting 1 ionizable function and a log $\mathrm{P}$ of -2.5 . Both peptides were paired with sodium docusate an anionic surfactant widely reported for the formation of HIP with peptides and proteins (Ristroph and Prud'homme, 2019). Using molar ratios of 1:2 leuprolide:docusate and 1:1.5 leuprolide:desmopressin, as described by Griesser et al. (Griesser et al., 2017), lead to precipitation efficiencies of $99.9 \pm 0.1 \%(\mathrm{n}=118)$ and $95.0 \pm 0.3 \%(\mathrm{n}=30)$ respectively, indicating that the majority of the peptides were in a lipidized state after this operation (Dumont et al., 2019b).

\subsection{Formulation of solid lipid nanocarriers using a scalable method}

\subsubsection{Material}

Solid lipid nanosuspensions are generally composed of 5 to $10 \%$ solid lipid excipient and 2 to $5 \%$ surfactants (Beloqui et al., 2016). In the case of NLC, a liquid lipid fraction representing 0.1 to $30 \%$ of the lipid content is added to the formulation.

In the present study, the solid lipid excipient used was Precirol ATO5 which is a mixture of palmitic and stearic glycerides obtained by esterification of glycerol and palmitic and stearic acids, with a melting point of $60^{\circ} \mathrm{C}$. The final product is a powder obtained by atomization. To formulate NLC, Capryol 190 , a monocaprylate ester of propylene glycol, was used as liquid lipid fraction. Several trials lead to the selection of the surfactant, Kolliphor RH40, polyoxyl-40 hydrogenated castor oil, which was used both in SLN and NLC (Dumont et al., 2019b).

\subsubsection{Process}

A broad bibliographic review was done to list the possible techniques to formulate solid lipid nanocarriers (Dumont et al., 2018). Among them, hot High Pressure Homogenization (HPH) technique was selected as it is a solvent-free and easily scalable process in the pharmaceutical industry. This method consists in the formation of an emulsion at a temperature higher than the melting point of the solid lipid excipient. This emulsion is then poured in the jacketed reservoir of a highpressure homogenizer where it is injected under high pressure in a chamber of interaction into which the oily droplets are disrupted against the walls leading to a drastic reduction of their size. Several cycles into the chamber are generally required to reach the nanoscale with a low polydispersity index. The final particles are obtained by cooling the nanoemulsion.

The process developed by our group is schematized in Figure 1. Briefly, the lipidized peptides were added in the lipid fraction melted at $70^{\circ} \mathrm{C}$. The emulsion was formed by addition of the surfactant solution heated at the same temperature and homogenization by Ultraturax during 3 minutes at $11000 \mathrm{rpm}$. The resulting emulsion was poured into the jacketed reservoir of a Microfluidics LM20 where it was submitted to 5 cycles at 500 bar. The resulting emulsion was cooled at $4{ }^{\circ} \mathrm{C}$ under magnetic stirring (Dumont et al., 2019b). Peptide solutions were submitted to the same process in absence of excipients and analyzed by HPLC to guarantee the absence of degradation of the drugs.

The formulations used in the rest of the study were composed of $68 \%$ Precirol $^{\circledR}$ ATO5, 5\% Capryol 90 and $27 \%$ Kolliphor ${ }^{\circledR} H 40$.

\subsection{Physico-chemical characterization of the formulations}

\subsubsection{Encapsulation efficiency}

The encapsulation efficiency (EE) of the nanoparticles was calculated as the difference between the total peptide concentration and the concentration of non-encapsulated peptide. The results showed that EE of $10.7 \pm 2.2 \%$ and $84.7 \pm 2.0 \%$ were reached when HIP of Desmopressin and Leuprolide were respectively encapsulated in NLC (Dumont et al., 2019b). The gap between the results was explained by the difference in $\log$ P value between HIP of desmopressin and leuprolide with an increase from -2.5 to +0.5 for desmopressin and from -2 to +3 for leuprolide when forming the ionic complex with docusate (Griesser et al., 2017). For the rest of the study only leuprolide was kept as drug model to evaluate the potential of solid lipid nanocarriers to increase the oral bioavailability of a model peptide. 


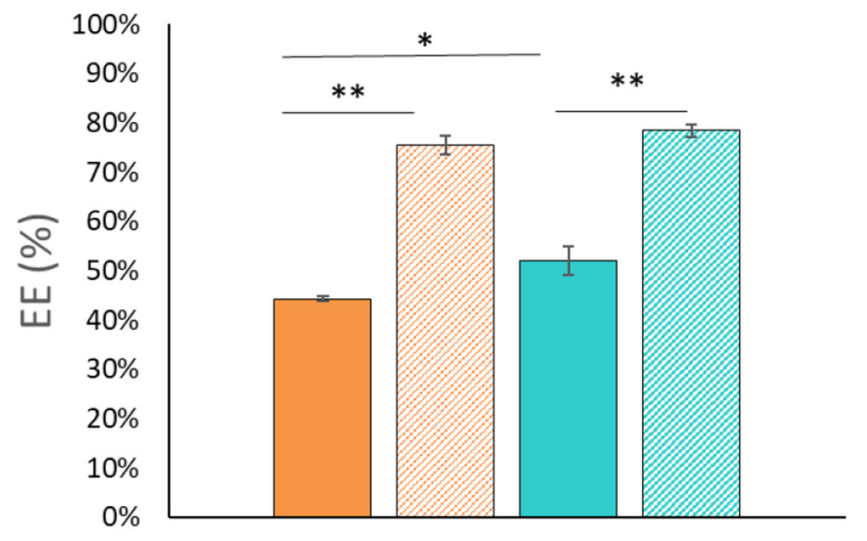

$\square$ NLCLEU $\quad$ NLCHIP $\square$ SLN LEU $\square$ SLN HIP

Fig. 2. Encapsulation efficiency of HIP or LEU as a function of the nanocarrier type $\left({ }^{*} p<0.05,{ }^{*} p<0.01\right)($ Mean \pm SEM, $\mathrm{n}=3)$.

NLC: nanostructured lipid carriers; LEU: leuprolide; HIP: hydrophobic ion pair; SLN: solid lipid nanoparticles.

The impact of the lipidization step on the loading of the peptide in the solid lipid nanocarriers was evaluated by measuring the EE of both leuprolide and HIP leuprolide: docusate in the designed SLN and NLC. The results showed a very significant difference between the EE of leuprolide and of the HIP in both type of nanocarriers which highlights the importance the importance of modifying peptides lipophilicity to boost their encapsulation in SLN and NLC (Fig. 2).

\subsubsection{Particle size}

It was shown that the permeation rate of self-emulsifying drug delivery systems across mucus was influenced by the size of the nanocarriers with an increased transport of small particles (below $200 \mathrm{~nm}$ ). The particle size distribution of the formulated nanosuspensions was characterized by Dynamic Light Scattering. All the formulations presented a particle size distribution below $130 \mathrm{~nm}$ with a polydispersity index of 0.2 (Tab. 1) (Dumont et al., 2019b). Neither did the addition of a liquid lipid fraction modified the particle size distribution between SLN and NLC, nor the loading of the peptide.

\subsubsection{Morphology}

Cryo-transmission electron microscopy (TEM) observations were conducted to confirm particle size distribution by direct observation of the nanocarriers. The images confirmed that the size of the nanoparticles was below $200 \mathrm{~nm}$ (Fig. 3). They also revealed the platelet-shape of the nanocarriers, which may be an asset in enhancing intestinal permeation, as it is the case for platelet-shaped polymeric nanoparticles (Banerjee et al., 2016).

\section{Results - Evaluation of systems behavior in intestinal environment}

\subsection{Protection towards proteases}

One of the main challenges in oral delivery of peptides is to provide a protective environment from the enzymatic degradation induced by proteases. Our systems were tested in the presence of trypsin and $\alpha$-chymotrypsin. Indeed, a rapid degradation of neat leuprolide was observed in the presence of trypsin (more than $80 \%$ degraded within 90 minutes) and $\alpha$-chymotrypsin (more than $80 \%$ degraded within 60 minutes) (Fig. 4). Although SLN did not provide a sheltering environment to leuprolide in the presence of both proteases, a protective effect from NLC against trypsin was observed, which was significant when leuprolide was encapsulated as a HIP (Dumont et al., 2019a). The protective effect generated by NLC can be explained by the presence of the liquid lipid fraction into which the proteases are not soluble, preventing their penetration into the carriers and access to the encapsulated peptide (Hetényi et al., 2017).

\subsection{Intestinal permeation}

The second major aspect limiting peptide oral bioavailability is their poor intestinal permeation. The potential of our lipid formulation in permeating the intestinal mucus and epithelium and increase peptide absorption was evaluated using Caco- 2 cell monolayers (enterocyte-like model) and Caco-2.HT29-MTX (goblet cells) co-cultures (mucus secreting model), as represented in Figure 5.

Internalization of the nanocarriers was observed by confocal light scanning microscopy (CLSM) after staining the cell monolayers co-incubated with fluorescently labelled SLN and NLC. The images showed a strong internalization of the carriers within both cell monolayers, indicating the capacity of the designed nanoparticles to be internalized by cells and to permeate the mucus layer at the surface of Caco-2/ HT29-MTX cell monolayers (Fig. 6). Fluorescence Activated Cell Sorting (FACS) was used to confirm the phenomenon, with $82 \%$ Caco-2 cells having internalized SLN and $99 \%$ of Caco-2 cells having internalized NLC (Dumont et al., 2020).

Given the encouraging permeation results obtained with the nanocarriers, their ability to increase leuprolide transport across the cell monolayers was evaluated by quantifying the peptide on the basolateral side of the cells. The results, displayed in Figure 7, showed that none of the carriers were able to boost the transport of leuprolide.

The explanation to these results was found by conducting drug release studies in HBSS (medium used for the permeation evaluation). The results showed that $90 \%$ on the peptide was released as soon as the nanosuspensions were diluted in HBSS medium. Indeed, this medium exhibits a high ionic strength with many ions able to disrupt the ionic bonding between leuprolide and docusate (Chamieh et al., 2019). Consequently, the peptide loses its hydrophobic characteristics and is more easily released from the particles. Furthermore, the plateletshaped structure of the nanocarriers implies a high interface between the particles and the external environment which negatively impacts the stability of the drug in the systems (Dumont et al., 2019a, 2020).

\section{Conclusions}

This study has demonstrated the possibility to encapsulate hydrophilic peptides in solid lipid nanocarriers using a solventfree and scalable process. It has shown that a lipidization step, 
Table 1. Particle size distribution of blank and peptide loaded SLN and NL. Reprinted from Dumont et al. (2019a) with permission from Elsevier.

\begin{tabular}{lllllll}
\hline & Blank NLC & NLC-LEU & NLC-HIP & Blank SLN & SLN-LEU & SLN-HIP \\
\hline Z-average (nm) & $114 \pm 11$ & $113 \pm 1$ & $125 \pm 2$ & $119 \pm 4$ & $124 \pm 1$ & $127 \pm 1$ \\
PDI & 0.2 & 0.2 & 0.2 & 0.2 & 0.2 & 0.2 \\
\hline
\end{tabular}

NLC: nanostructured lipid carriers; LEU: leuprolide; HIP: hydrophobic ion pair; SLN: solid lipid nanoparticles.
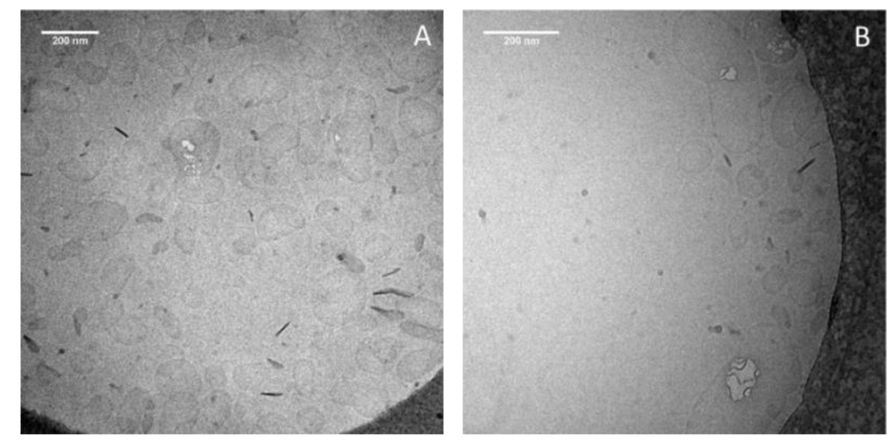

Fig. 3. Cryo-transmission electron microscopy (TEM) observation of (A) Precirol ATO5/Kolliphor RH40 SLN and (B) Precirol ${ }^{\circledR}$ ATO5/ Capryol $90 /$ Kolliphor ${ }^{\circledR}$ RH40 NLC obtained by hot high pressure homogenization (HPH) technique. Nanoparticles are ellipsoidal platelets with the darker rods corresponding to edge-on nanoparticles with an increased thickness. Reprinted from Dumont et al. (2019a) with permission from Elsevier.

Trupsin

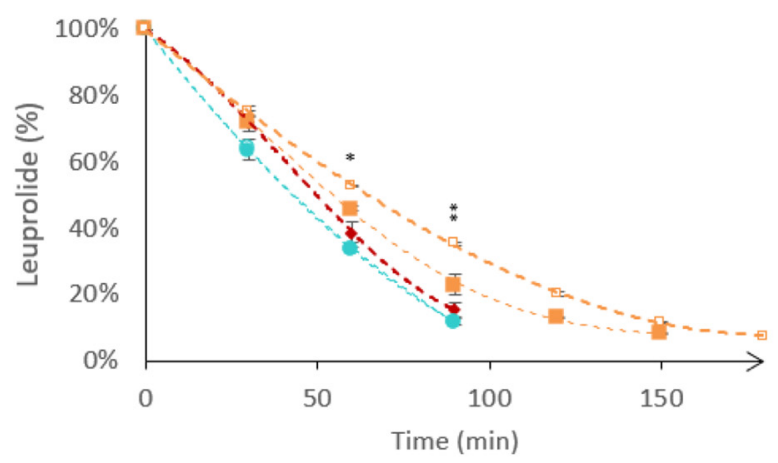

$\alpha$-chymotrypsin

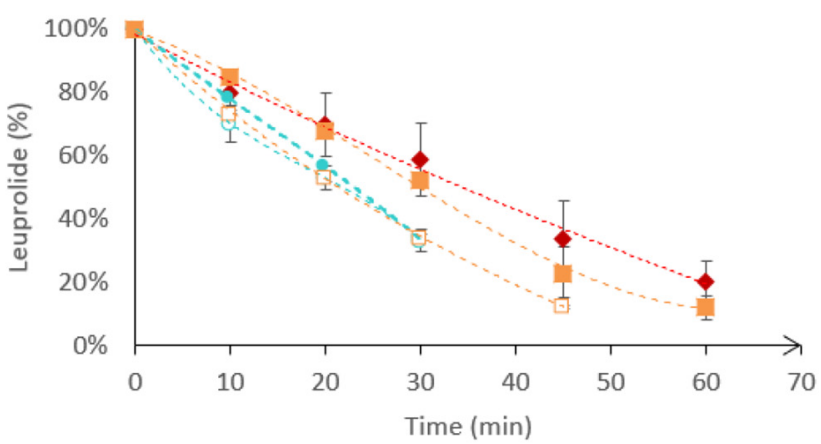

Fig. 4. Degradation profile of leuprolide in NLC LEU (fx1), NLC HIP (fx2), SLN LEU (fx3) and SLN HIP (fx4) by trypsin and $\alpha$-chymotrypsin compared to free LEU (fx5) in Tris-HCl pH 6.8 buffered medium (mean $\pm \mathrm{SEM}, \mathrm{n}=3,{ }^{*} p<0.05$ and ${ }^{*} p<0.01$ ). Reprinted from Dumont et al. (2019a) with permission from Elsevier.

NLC: nanostructured lipid carriers; LEU: leuprolide; HIP: hydrophobic ion pair; SLN: solid lipid nanoparticles.

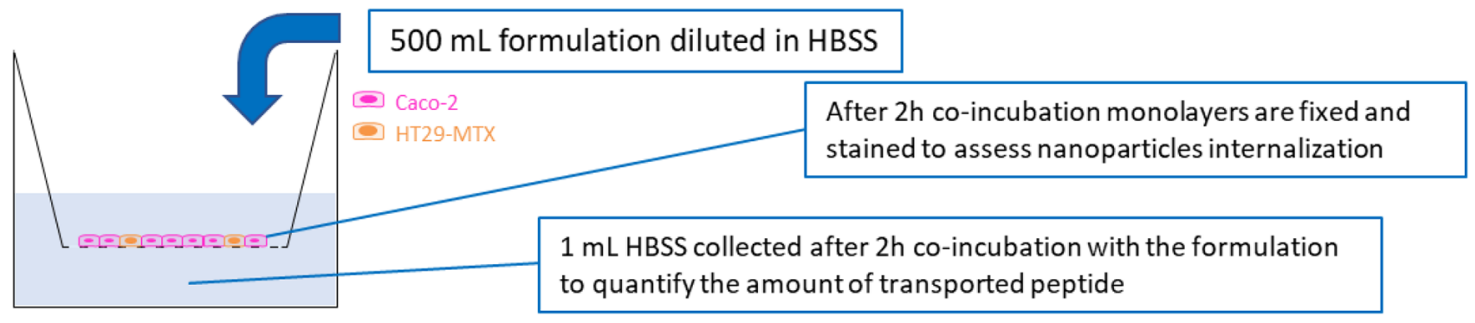

Fig. 5. In vitro cell monolayers to evaluate the permeation of nanocarriers and encapsulated peptide. 


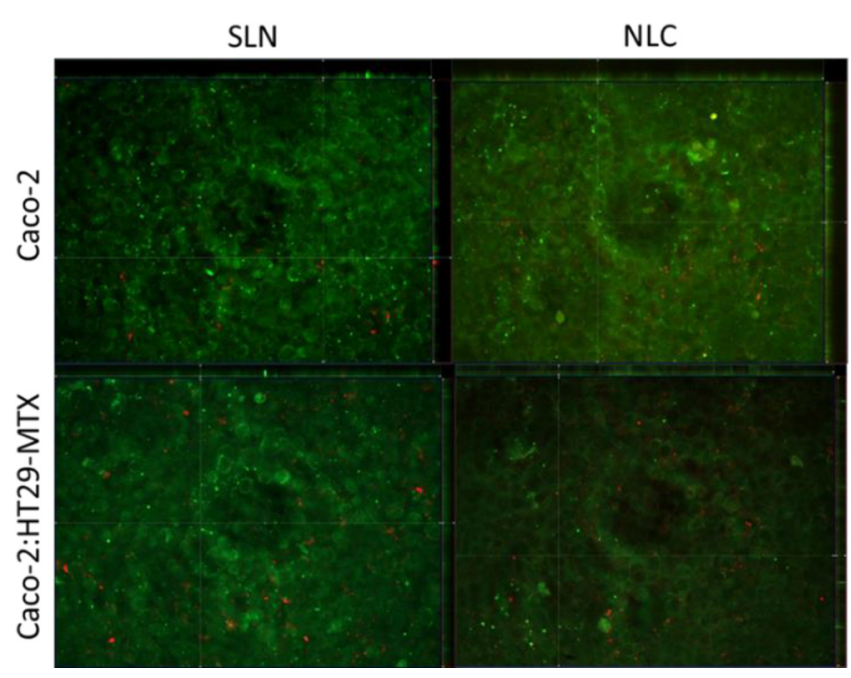

Fig. 6. Confocal light scanning microscopy (CLSM) images of SLN and NLC with Caco-2 and Caco-2/HT29-MTX co-cultured cells monolayers after $2 \mathrm{~h}$ incubation at $37^{\circ} \mathrm{C}(\mathrm{x} 25)$. The green channel indicates rhodamine-phalloidin and the DiD-loaded nanoparticles are indicated by the red channel. Reprinted from Dumont et al. (2020) with permission from Elsevier.

SLN: solid lipid nanoparticles; NLC: nanostructured lipid carriers.

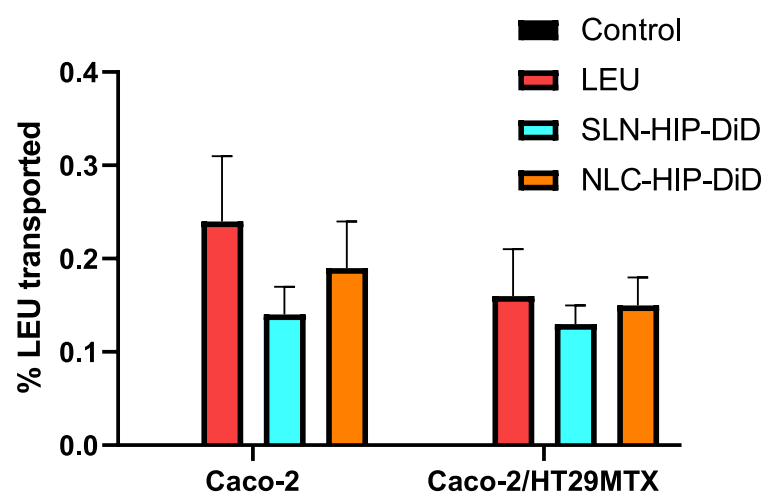

Fig. 7. Percentage of transported leuprolide as a function of nanocarrier and cell monolayer.

LEU: leuprolide; SLN: solid lipid nanoparticles; NLC: nanostructured lipid carriers; HIP: hydrophobic ion pair.

such as the formation of Hydrophobic Ion Pairs, is required to boost the encapsulation efficiencies of peptides in solid lipid nanoparticles and nanostructured lipid carriers.

The designed nanoparticles presented a particle size below $200 \mathrm{~nm}$ and a platelet morphology. NLC were able to protect encapsulated peptides from proteolytic degradation induced by trypsin. Both nanocarrier types were highly internalized by intestinal epithelium cell models and were able to cross the mucus layer. However, the instability of the hydrophobic ion pair associated with the platelet-shape of the carriers limited the intestinal transport of the peptide.

In conclusion: solid lipid nanocarriers are a promising strategy to enhance the oral bioavailability to encapsulated peptide. However, further adjustments such as a modification of the particles shape and a stabilization of the peptide hydrophobic ion pair are necessary to fully benefit from these nanocarriers.

Acknowledgements. This study was supported by Gattefossé SAS via a CIFRE contract between Gattefossé SAS and LAGEPP (University Claude Bernard Lyon 1). The permeation studies were performed during a Short-Term Scientific Mission between Gattefossé SAS/Lyon University and Louvain Drug Research Institute at the UC Louvain and received funding from COST Action 16205 UNGAP.

\section{References}

Aguirre TAS, Teijeiro-Osorio D, Rosa M, Coulter IS, Alonso MJ, Brayden DJ. 2016. Current status of selected oral peptide technologies in advanced preclinical development and in clinical trials. Adv Drug Deliv Rev 106: 223-241.

Banerjee A, Qi J, Gogoi R, Wong J, Mitragotri S. 2016. Role of nanoparticle size, shape and surface chemistry in oral drug delivery. J Control Release: Off J Control Release Soc 238: 176185.

Beloqui A, Solinís MÁ, Rodríguez-Gascón A, Almeida AJ, Préat V. 2016. Nanostructured lipid carriers: Promising drug delivery systems for future clinics. Nanomed: Nanotechnol Biol Med 12: 143-161.

Chamieh J, Domènech Tarrat A, Doudou C, Jannin V, Demarne F, Cottet H. 2019. Peptide release from SEDDS containing hydrophobic ion pair therapeutic peptides measured by Taylor dispersion analysis. Int J Pharm 559: 228-234.

Craik DJ, Fairlie DP, Liras S, Price D. 2013. The future of peptidebased drugs. Chem Biol Drug Des 81: 136-147.

Dumont C, Bourgeois S, Fessi H, Jannin V. 2018. Lipid-based nanosuspensions for oral delivery of peptides, a critical review. Int $J$ Pharm 541: 117-135.

Dumont C, Bourgeois S, Fessi H, Dugas P-Y, Jannin V. 2019a. Invitro evaluation of solid lipid nanoparticles: Ability to encapsulate, release and ensure effective protection of peptides in the gastrointestinal tract. Int J Pharm 565: 409-418.

Dumont C, Jannin V, Miolane C, et al. 2019b. A proof-of-concept for developing oral lipidized peptide nanostructured lipid carrier formulations. J Drug Deliv Sci Technol 54: 101394.

Dumont C, Beloqui A, Miolane C, et al. 2020. Solid lipid nanocarriers diffuse effectively through mucus and enter intestinal cells - But where is my peptide? Int J Pharm 586: 119581.

Griesser J, Hetényi G, Kadas H, Demarne F, Jannin V, BernkopSchnürch A. 2018. Self-emulsifying peptide drug delivery systems: How to make them highly mucus permeating. Int J Pharm 538: 159-166.

Griesser J, Hetényi G, Moser M, Demarne F, Jannin V, BernkopSchnürch A. 2017. Hydrophobic ion pairing: Key to highly payloaded self-emulsifying peptide drug delivery systems. Int J Pharm 520: 267-274.

Hetényi G, Griesser J, Moser M, Demarne F, Jannin V, BernkopSchnürch A. 2017. Comparison of the protective effect of selfemulsifying peptide drug delivery systems towards intestinal proteases and glutathione. Int J Pharm 523: 357-365.

Leonaviciute G, Bernkop-Schnürch A. 2015. Self-emulsifying drug delivery systems in oral (poly)peptide drug delivery. Exp Opin Drug Deliv 12: 1703-1716.

Malhaire H, Gimel J-C, Roger E, Benoît J-P, Lagarce F. 2016. How to design the surface of peptide-loaded nanoparticles for efficient oral bioavailability? Adv Drug Deliv Rev 106: 320-336. 
Morishita M, Peppas NA. 2006. Is the oral route possible for peptide and protein drug delivery? Drug Discov Today 11: 905-910.

Müller RH. 2011. 20 years of lipid nanoparticles (SLN \& NLC): Present state of development \& industrial applications. Curr Drug Discov Technol 8: 207-27.
Richard J. 2017. Challenges in oral peptide delivery: Lessons learnt from the clinic and future prospects. Ther Deliv 8: 663-684. Ristroph KD, Prud'homme RK. 2019. Hydrophobic ion pairing: Encapsulating small molecules, peptides, and proteins into nanocarriers. Nanoscale $A d v$ 1: 4207-4237.

Cite this article as: Dumont C. 2022. Lipid-based nanocarriers for oral delivery of peptides. OCL 29: 1. 